\title{
Cuestionarios de control de asma pediátrica y calidad de vida
}

\author{
ALBERTO VIDAL G. ${ }^{1}$ \\ 1. Pediatra, Especialista en Enfermedades Respiratorias, Departamento de Pediatría, Clínica Las Condes.
}

\begin{abstract}
Pediatric asthma control and quality of life questionnaires

The assessment of symptoms and quality of life are important aspects in the control of childhood asthma. Questionnaires regarding symptoms control and quality of life give valuable information about the disease from the perspective of the child and his family. The aim of this study is to discuss the most important characteristics of this type of questionnaire such as age range, language of validation, number of questions, administration mode, the evaluated period and fields, and the minimum clinically relevant difference. The ACT questionnaires, for children older than 11 years of age, and CAN questionnaires, for children between 2 and 14 years of age in their Spanish versions, are suitable for measuring asthma control in our country. Regarding quality of life, PAQLQ for children between 7 and 17 years of age and PACQLQ for their care givers are the most popular oversees; Spanish versions have been adapted for Chile.
\end{abstract}

(Key words: Asthma, quality of life, children, teenagers).

Rev Chil Pediatr 2014; 85 (3): 359-366

\section{RESUMEN}

La evaluación de los síntomas y la calidad de vida son aspectos importantes en el control del asma infantil. Los cuestionarios de control de síntomas y de calidad de vida entregan información de la enfermedad desde la perspectiva del niño y su familia. El objetivo de esta revisión es discutir las características más importantes de este tipo de cuestionarios incluyendo el rango de edad, el idioma de validación, el número de preguntas, el modo de administración, el período evaluado, los dominios evaluados y la diferencia mínima clínicamente relevante. Los cuestionarios ACT para niños mayores de 11 años y CAN para niños entre 2 y 14 años en sus versiones en español, son apropiados para medir el control del asma en nuestro país. En calidad de vida, el PAQLQ para niños de 7 a 17 años y PACQLQ para sus cuidadores, son los más difundidos internacionalmente, con versiones en español adaptadas para Chile.

(Palabras clave: Asma, calidad de vida, niños, adolescentes).

Rev Chil Pediatr 2014; 85 (3): 359-366

Recibido el 26 de febrero de 2012, devuelto para corregir el 10 de abril de 2013, segunda versión 26 de julio de 2013, tercera versión 18 de noviembre de 2013, aceptado para publicación el 28 de enero de 2014.

Este trabajo cumple con los requisitos sobre consentimiento /asentimiento informado, comité de ética, financiamiento, estudios animales y sobre la ausencia de conflictos de intereses según corresponda.

Correspondencia a:

Alberto Vidal G.

E-mail: aevgmd@yahoo.es 


\section{Introducción}

El control del asma en los niños se ha definido como el grado en que se pueden reducir o eliminar las manifestaciones del asma con un adecuado tratamiento. En la actualidad se recomienda medirlo teniendo en consideración algunos aspectos como: la medición del control actual por medio de la evaluación de síntomas, la normalidad en las actividades cotidianas, la calidad de vida, el riesgo futuro de eventos adversos como las exacerbaciones, el deterioro de la función pulmonar y los efectos del tratamiento ${ }^{1}$.

Las guías internacionales de manejo del asma recomiendan monitorizar el control del asma pediátrica por medio de la evaluación de los síntomas, la calidad de vida, la función pulmonar y algunos marcadores inflamatorios ${ }^{2,3}$.

No existe un método ideal para la monitorización del control de asma en los niños, cada uno tiene fortalezas y debilidades, sin embargo, la irrupción de los cuestionarios de control y de calidad de vida han permitido conocer el impacto del asma desde la perspectiva del niño y de su familia ${ }^{4}$.

En las últimas dos décadas se han desarrollado y validado numerosos cuestionarios de control y calidad de vida en asma. Los más importantes han sido publicados en diferentes revistas de literatura médica, cumpliendo con los requisitos para un estudio de validación.

El presente estudio es una revisión bibliográfica que incluyó a todos los cuestionarios de control y calidad de vida en niños y adolescentes con al menos un trabajo de validación publicado. Se analizaron las características más importantes de estos cuestionarios con el objetivo de identificar a aquellos recomendables o factibles de aplicar en nuestro medio.

\section{Cuestionarios de control de síntomas}

El control del asma es un constructo multidimensional que mide el nivel alcanzado de la meta propuesta de tratamiento. Los cuestionarios utilizados para medirlo son instrumentos capaces de evaluar la actividad crónica de del asma en períodos que por lo general van desde
1 semana a un mes. La mayoría son autoadministrables y se aplican mediante entrevistas en visitas clínicas en el lugar de atención médica o menos comúnmente, a través de versiones electrónicas para ser respondidos en el hogar ${ }^{5}$.

Aunque no hay consenso con respecto a que área evaluar, casi todos incluyen preguntas acerca de síntomas diurnos, síntomas nocturnos o alteraciones del sueño y limitaciones de la actividad cotidiana provocadas por la enfermedad. Algunos cuestionarios han incorporado preguntas en relación al uso de medicamentos de rescate como los broncodilatadores, número de exacerbaciones o crisis, hospitalizaciones e incluso valores de función pulmonar. En la mayoría de los cuestionarios las alternativas de respuesta se ordenan en escalas ordinales de Likert que pueden ser ascendentes o descendentes, permitiendo obtener un puntaje promedio con el que finalmente se le asigna al paciente el grado de control del asma bronquial ${ }^{6}$.

Existen más de una decena de cuestionarios para medir el control del asma pediátrica que se han publicado en los últimos años. Bateman et al, fueron los primeros en evaluar retrospectivamente varios estudios clínicos para identificar y validar las preguntas más utilizadas en la medición de control en asma para pacientes mayores de 12 años y adultos ${ }^{7,8}$. La Iniciativa Global para el Asma (GINA) recogió las recomendaciones de estos expertos y actualmente recomienda un cuestionario de control para niños mayores de 5 años que entrega tres niveles de cualitativos de control: controlado, parcialmente controlado y no controlado ${ }^{9}$. La versión en español del cuestionario GINA no tiene estudios de validación, sin embargo, es utilizado por muchas unidades de enfermedades respiratorias pediátricas de Latinoamérica, incluyendo a las de nuestro país.

De los cuestionarios de control validados, los más difundidos internacionalmente son cuatro: el cuestionario ACT (Asthma Control Test), el C-ACT (Childhood Asthma Control Test), ACQ (Asthma Control Questionnaire) y el C-ATAQ (Children Asthma Therapy Assessment Questionnaire).

El cuestionario ACT fue diseñado para asmáticos desde los 12 años y adultos y permi- 
te obtener un puntaje total que tiene un rango desde 5 (peor control) a 25 puntos (control total). Los pacientes con puntaje total menor a 20 puntos se clasifican como no controlados y aquellos con puntaje menor a 16 puntos son categorizados como pobremente controlados ${ }^{10,11}$. Este cuestionario se ha validado en español y también ha sido replicado en nuestro medio, logrando medir con éxito el control de asma en adolescentes de 12 o más años ${ }^{12,13}$.

El C-ACT es la versión de ACT para niños menores de 12 años. Este cuestionario integra las respuestas del niño y de su cuidador, para obtener un puntaje total de 0 a 27 puntos, considerando asma no controlada a aquella con menos de 20 puntos, sin embargo, sólo está validado en inglés ${ }^{14}$.

El ACQ de Juniper es un cuestionario recientemente validado en inglés, que además de las preguntas de control, incorpora la medición del $\mathrm{VEF}_{1}$. Un puntaje mayor o igual a 1,5 puntos sugiere asma fuera de control y la recomendación para los asmáticos menores de 10 años es que sea aplicado por un entrevistador entrenado ${ }^{15}$.

El C-ATAQ es un cuestionario validado en inglés con preguntas dirigidas a los padres o cuidadores. Los promedios mayores o igual a 1 indican asma no bien controlada que requiere consulta al médico tratante ${ }^{16,17}$.

Existen dos cuestionarios emergentes: el CAN (cuestionario de control de asma en niños), diseñado y validado en español, por un grupo de especialistas españoles y el TRACK (test for Respiratory and Asthma Control in Kidz) que es uno de los pocos cuestionarios de asma específicos para preescolares.

CAN cuenta con dos versiones, una para niños de 2 a 8 años con preguntas que deben ser respondidas por el cuidador y otra para niños o adolescentes de 9 hasta 14 años, que pueden ser respondidas por el paciente o su cuidador. Se obtiene un puntaje total que va desde 0 (ausencia de síntomas) hasta 36 puntos (máxima sintomatología) y se consideran sin control a los asmáticos con puntaje promedio total igual o superior a 8 puntos ${ }^{18}$.

El TRACK es un cuestionario para cuidadores validado en inglés y sus respuestas entregan un puntaje promedio de 0 (máxima sintomatología) a 100 puntos (ausencia de sintomatología). Los niños menores de 5 años con menos de 80 puntos en este cuestionario se consideran controlados ${ }^{19,20}$.

Existen otros cuestionarios de control de asma menos conocidos, que tienen al menos un estudio de validación en niños o adolescentes de diferentes edades. En la tabla 1 se muestran las características más importantes de los cuestionarios de control de asma: rango de edad para el cual fueron validados, número de preguntas utilizadas, el período que evalúa y el modo de administración. Además se destacan los que cuentan con validación en español y aquellos que incluyen dentro de sus pruebas psicométricas la diferencia mínima clínicamente relevante, que corresponde a la diferencia más pequeña en la puntuación de un dominio que es percibida como beneficiosa por los pacientes y que justifica un cambio del manejo clínico.

\section{Cuestionarios de calidad de vida}

La calidad de vida en asma mide el impacto que la enfermedad o su tratamiento tiene en áreas como la salud física, psicosocial y espiritual de la persona que padece la enfermedad. Se mide a través de instrumentos que llevan a puntajes los juicios de valor que los pacientes asmáticos son capaces de elaborar respecto de su enfermedad. Se utilizan en asma con diferentes objetivos: describir el efecto de la enfermedad en el paciente, control de la terapia farmacológica y estudios clínicos o de análisis costo-beneficio en el tratamiento.

Los cuestionarios de calidad de vida en niños y adolescentes asmáticos tienen algunas consideraciones especiales que deben ser mencionadas. La edad es un elemento a tener en cuenta, ya que se ha demostrado que los niños mayores de 10 años son capaces de elaborar el concepto de la enfermedad crónica y el impacto que esta les genera en su vida, sin embargo, los niños menores de 6 años tienen dificultad para entender conceptos abstractos como lo relacionado con la emocionalidad o las limitaciones en área social.

Por otra parte, las áreas evaluadas deben 
Tabla 1. Características de los cuestionarios de control de asma para niños y adolescentes

\begin{tabular}{|c|c|c|c|c|c|c|}
\hline Cuestionario & $\begin{array}{l}\text { Rango de } \\
\text { edad (años) }\end{array}$ & $\begin{array}{l}\text { Idioma de } \\
\text { validación }\end{array}$ & $\begin{array}{c}\mathrm{n} \text { de } \\
\text { preguntas }\end{array}$ & $\begin{array}{l}\text { Período } \\
\text { evaluado }\end{array}$ & $\begin{array}{c}\text { Modo de } \\
\text { administración }\end{array}$ & $\begin{array}{c}\text { DMCR } \\
\text { (puntos) }\end{array}$ \\
\hline $\mathrm{ACT}^{10}$ & $\geq 12$ & 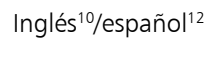 & 5 & 4 semanas & $\begin{array}{l}\text { Autoadministrable escrito } \\
\text { al paciente }\end{array}$ & 3 \\
\hline C-ACT 14 & 4 a 11 & Inglés & 7 & 4 semanas & $\begin{array}{l}\text { Autoadministrable escrito } \\
\text { al paciente y tutor }\end{array}$ & No \\
\hline $\mathrm{ACQ}^{15}$ & 6 a 16 & Inglés & 7 & 1 semana & $\begin{array}{l}\text { Autoadministrable escrito } \\
\text { al paciente ( } 10 \text { a } 16 \text { años) y } \\
\text { tutor ( } 6 \text { a } 16 \text { años) }\end{array}$ & 0,5 \\
\hline C-ATAQ $^{16,17}$ & 5 a 17 & Inglés & 7 & 1 a 12 meses & Entrevista al tutor & No \\
\hline CAN $^{18}$ & 2 a 14 & Español ${ }^{18}$ & 9 & 4 semanas & $\begin{array}{l}\text { Autoadministrable escrito } \\
\text { al paciente ( } 9 \text { a } 14 \text { años) o } \\
\text { tutor ( } 2 \text { a } 14 \text { años) }\end{array}$ & No \\
\hline Track ${ }^{19,20}$ & 0 a 5 & Inglés & 5 & 4 semanas & $\begin{array}{l}\text { Autoadministrable escrito } \\
\text { al tutor }\end{array}$ & No \\
\hline Quiz'21 & 1 a 17 & Inglés & 6 & 4 semanas & $\begin{array}{l}\text { Autoadministrable escrito } \\
\text { al paciente (9 a } 17 \text { años) o } \\
\text { tutor (1 a } 17 \text { años) }\end{array}$ & No \\
\hline $\mathrm{PACT}^{22}$ & 1 a 18 & Inglés & 10 & 3 meses & $\begin{array}{l}\text { Autoadministrable escrito } \\
\text { al tutor }\end{array}$ & No \\
\hline Breathmobile 23 & 2 a 14 & Inglés & 7 & 1 a 24 meses & $\begin{array}{l}\text { Autoadministrable escrito } \\
\text { al tutor }\end{array}$ & No \\
\hline FSAS $^{24}$ & 3 a 17 & Inglés & 6 & 12 meses & $\begin{array}{l}\text { Autoadministrable escrito } \\
\text { al tutor }\end{array}$ & No \\
\hline LASS 25 & 3 a 17 & Inglés & 8 & 4 semanas & $\begin{array}{l}\text { Autoadministrable escrito } \\
\text { al paciente o tutor }\end{array}$ & 7 \\
\hline SASCQ $^{26}$ & $\geq 12$ & Inglés & 5 & 4 semanas & $\begin{array}{l}\text { Autoadministrable escrito } \\
\text { al paciente }\end{array}$ & No \\
\hline RCP27 & 6 a 15 & Inglés & 3 & $\begin{array}{l}1 \text { semana a } \\
1 \text { mes }\end{array}$ & $\begin{array}{l}\text { Autoadministrable escrito } \\
\text { al paciente }\end{array}$ & No \\
\hline
\end{tabular}

DMCR: Diferencia mínima clìnicamente relevante, ACT: Asthma Control Test, C-ACT: Child Asthma Control Test, ACQ: Asthma Control Questionnaire, C-ATAQ: Asthma Terapy Assesment Questionnaire for children and adolescents, CAN: Cuestionario de control de asma en niños, Track: Test for Respiratory and Asthma Control in Kids, Quiz: Asthma Quis for Kidz, PACT: Pediatric Asthma Control Tool, Breathmobile: Breathmobile Assessment of Asthma Control, FSAS: Functional severity of asthma Scale, LASS: Lara Asthma Symptom Scale, SASCQ: Seattle Asthma Severity and Control Questionnaire, RCP: Royal College of Physicians.

considerar la etapa del niño, siendo más importantes para los niños preescolares y escolares los dominios de síntomas o de limitación de actividades, que en los adolescentes, en los cuales son más útiles los dominios sociales y emocionales.

Otro aspecto importante es el grado de acuerdo entre la percepción de calidad de vida del niño y la de su cuidador, ya que la concordancia entre estos depende del dominio evaluado. Se sabe que se obtiene mejor grado de acuerdo al evaluar los síntomas y el funcionamiento físico, que en las áreas sociales o emocionales $^{28}$.
Por último, la capacidad de atención en niños pequeños es menor que en los adolescentes, siendo recomendable no utilizar más de 3 o 4 opciones de respuesta, que incluyan escalas visuales análogas con ilustraciones graduadas por tamaño o por expresión facial. Además en niños pequeños se debe promover el uso de cuestionarios aplicados por un entrevistador amigable que cuente con entrenamiento adecuado, a diferencia de los adolescentes que no tienen dificultades para responder los cuestionarios auto-administrables ${ }^{29}$.

Los cuestionarios más conocidos son el PAQLQ (Paediatric Asthma Quality of Life 
Questionnaire) y el PACQLQ (Paediatric Asthma Caregiver's Quality of Life Questionnaire) ambos validados por Elizabeth Juniper en 1996. Se responden a través de una escala ordinal de Likert, por medio de la cual se obtiene un puntaje de calidad de vida. Se ha demostrado su utilidad en el seguimiento de la calidad de vida del niño asmático o su cuidador y también en estudios de investigación. Una diferencia o cambio mayor o igual a 0,5 puntos en el puntaje de calidad de vida es considera clínicamente relevante en estos cuestionarios $^{30,31}$. Ambos cuestionarios tienen traducciones validadas en diferentes idiomas, incluso versiones validadas para nuestro país, las cuales se han aplicado sin dificultad en niños asmáticos y en cuidadotes, atendidos por médicos de la atención primaria y por pediatras especialistas en enfermedades respiratorias $^{32-35}$

La Academia Americana de Pediatría ha desarrollado un cuestionario específico de calidad de vida que infiere la calidad de vida del niño por medio de la percepción obtenida del cuidador, llamado CHSA (Children's Health Survey for Asthma) y otra versión para ser aplicada directamente al niño llamada CHSAC (Children's Health Survey for Asthma-Child Version). Ambas deben ser respondidas en una escala ordinal de Likert ascendente de 0 (peor calidad de vida) a 100 puntos (mejor calidad de vida). CHSA tiene la fortaleza de incluir la evaluación de los dominios de actividad y emocionalidad en el grupo familiar, sin embargo, es demasiado extenso como para ser implementado en los controles de rutina, resultando mucho más útil en investigación ${ }^{36-38}$.

Otro cuestionario conocido es el PedsQLAM 3.0 (Pediatric Quality of Life Inventory Asthma Module 3.0), que se responde por medio de una escala de Likert reversa de 5 opciones para los niños y adolescentes de 8 a 18 años y tres opciones para niños de 5 a 7 años. El mayor puntaje obtenido por este cuestionario indica mejor calidad de vida. Tiene tres versiones auto administrables para padres o niños (5-7 años, 8-12 años, 13-18 años) y una versión para preescolares de 2 a 4 años que se aplica sólo a los padres ${ }^{39-41}$.

Dos cuestionarios abreviados se han publi- cado en los últimos años. Uno es el PAQLQ ilustrado (pictorial PAQLQ), que se construyó acortando la versión original del PAQLQ por medio de la eliminación del dominio de limitación de actividades. Es una buena alternativa para niños menores de 8 años, los cuales tienen menos concentración pudiendo entregar una información diferente a la de su cuidador. Se administra por medio de una entrevista al niño, quien debe marcar sus respuestas en una escala visual análoga de termómetros con diferentes niveles de temperatura, que representan la cantidad de síntomas o alteraciones presentes en el niño ${ }^{42}$. El otro es el MiniPAQLQ que disminuyó las preguntas del PAQLQ conservando los dominios, el método de administración y el rango de edad para ser aplicado, sin alterar validez y reproducibilidad de la versión original $^{43}$.

En la tabla 2 se describen algunas de las características importantes de los cuestionarios de calidad de vida en asma pediátrica: rango de edad, idioma de validación, número de preguntas, áreas o dominios evaluados, modo de administración y diferencia mínima clínicamente relevante.

\section{Conclusiones}

En las últimas décadas se han validado una gran cantidad de cuestionarios de control y calidad de vida en asma pediátrica. Casi todos están diseñados para ser implementados en formato autoadministrable al niño o a su cuidador, lo que los hace fáciles de aplicar. La correcta elección de un cuestionario debe considerar la edad del niño o adolescente, su grado de madurez, la extensión del cuestionario, las áreas o dominios que desean ser evaluados y el idioma de validación. La mayoría de los cuestionarios tienen validación original en inglés, lo que dificulta su utilización en nuestro país. Por sus propiedades psicométricas, extensión y lenguaje de validación, los cuestionarios ACT para niños mayores de 11 años y CAN para niños de 2 a 14 años son los más indicados para evaluar el control de asma en nuestro medio. Las versiones de ACT para niños de 4 a 11 años (C-ACT) y ACQ para niños de 6 a 16 
Tabla 2. Características de los cuestionarios de calidad de vida para niños y adolescentes con asma

\begin{tabular}{|c|c|c|c|c|c|c|}
\hline Cuestionario & $\begin{array}{l}\text { Rango } \\
\text { de edad } \\
\text { (años) }\end{array}$ & $\begin{array}{l}\text { Idioma de } \\
\text { validación }\end{array}$ & $\begin{array}{c}\mathrm{n} \text { de } \\
\text { preguntas }\end{array}$ & Areas o dominios & $\begin{array}{c}\text { Modo de } \\
\text { administración }\end{array}$ & $\begin{array}{c}\text { DMCR } \\
\text { (puntos) }\end{array}$ \\
\hline PAQLQ 30 & $7-17$ & $\begin{array}{l}\text { Inglés/ } \\
\text { españo| }{ }^{32}\end{array}$ & 23 & $\begin{array}{l}\text { Síntomas, limitación de acti- } \\
\text { vidades y función emocional }\end{array}$ & $\begin{array}{l}\text { Autoadministrable o entrevis- } \\
\text { ta al niño/adolescente }\end{array}$ & 0,5 \\
\hline PACQLQ 31 & $7-17$ & $\begin{array}{l}\text { Inglés/ } \\
\text { españo| }{ }^{32}\end{array}$ & 13 & $\begin{array}{l}\text { Función emocional y limita- } \\
\text { ción de actividades }\end{array}$ & Autoadministrable al cuidador & 0,5 \\
\hline $\mathrm{CHSA}^{36,37}$ & $5-12$ & Inglés & 48 & $\begin{array}{l}\text { Salud física, salud emocional } \\
\text { (niño y familia) y actividad } \\
\text { (niño y familia) }\end{array}$ & $\begin{array}{l}\text { Autoadministrable a cuida- } \\
\text { dores }\end{array}$ & No \\
\hline CHSA-C ${ }^{38}$ & $7-16$ & Inglés & 25 & $\begin{array}{l}\text { Salud física, actividades y } \\
\text { salud emocional }\end{array}$ & Entrevista al niño/adolescente & No \\
\hline $\begin{array}{l}\text { PedsQL-AM } \\
3.0^{39-41}\end{array}$ & $2-18$ & Inglés & 28 & $\begin{array}{l}\text { Síntomas, problemas del } \\
\text { tratamiento, comunicación y } \\
\text { preocupaciones }\end{array}$ & $\begin{array}{l}\text { Autoadministrable al cuidador } \\
\text { (niños de } 2-18 \text { años) } \\
\text { Autoadministrable a niños/ } \\
\text { adolescentes de } 5 \text { a } 18 \text { años) }\end{array}$ & No \\
\hline $\begin{array}{l}\text { Pictorial } \\
\text { PAQLQ }\end{array}$ & $5-7$ & Inglés & 15 & Síntomas y emociones & Entrevista a niño & No \\
\hline MiniPAQLQ ${ }^{43}$ & $7-17$ & Inglés & 13 & $\begin{array}{l}\text { Función emocional y limita- } \\
\text { ción de actividades }\end{array}$ & $\begin{array}{l}\text { Autoadministrable al niño/ } \\
\text { adolescente }\end{array}$ & No \\
\hline $\mathrm{AMA}^{44}$ & $6-12$ & Inglés & 55 & $\begin{array}{l}\text { Preguntas no divididas en } \\
\text { dominios }\end{array}$ & $\begin{array}{l}\text { Autoadministrable o entrevis- } \\
\text { ta al niño/adolescente }\end{array}$ & No \\
\hline $\mathrm{AAQOL}^{45}$ & $12-17$ & Inglés & 32 & $\begin{array}{l}\text { Síntomas, medicamentos, } \\
\text { actividad física, emociones, } \\
\text { interacción social y efectos } \\
\text { positivos }\end{array}$ & $\begin{array}{l}\text { Autoadministrable al ado- } \\
\text { lescente }\end{array}$ & No \\
\hline \multicolumn{7}{|l|}{$\mathrm{CAQ}^{46}$} \\
\hline CAQ A & $4-7$ & Inglés & 14 & Calidad de vida y estrés & Autoadministrable al niño & \multirow{3}{*}{ No } \\
\hline CAQ B & $8-11$ & & 22 & $\begin{array}{l}\text { Calidad de vida activa, pasiva, } \\
\text { severidad y estrés }\end{array}$ & Autoadministrable al niño & \\
\hline CAQ C & $12-16$ & & 31 & $\begin{array}{l}\text { Calidad de vida activa, pasiva, } \\
\text { severidad, estrés y reactividad }\end{array}$ & $\begin{array}{l}\text { Autoadministrable al ado- } \\
\text { lescente }\end{array}$ & \\
\hline ARQOLS 47 & $6-13$ & Inglés & 35 & $\begin{array}{l}\text { Alteración física, limitación } \\
\text { actividad física, restricción } \\
\text { social, inconvenientes diarios } \\
\text { en manejo de enfermedad o } \\
\text { estrés emocional }\end{array}$ & Entrevista al niño & No \\
\hline $\begin{array}{l}\text { TACQOL- } \\
\text { asthma }^{48}\end{array}$ & $8-16$ & Inglés & 68 & $\begin{array}{l}\text { Síntomas, gatillantes, visitas al } \\
\text { médico, uso de medicamen- } \\
\text { tos y emociones negativas }\end{array}$ & $\begin{array}{l}\text { Entrevista al niño/adolescente } \\
\text { y cuidador }\end{array}$ & No \\
\hline LAQCL $^{49}$ & $5-17$ & Inglés & 71 & $\begin{array}{l}\text { Función física, función emo- } \\
\text { cional, actividades escolares y } \\
\text { de la vida diaria }\end{array}$ & $\begin{array}{l}\text { Autoadministrable al niño/ } \\
\text { adolescente }\end{array}$ & No \\
\hline $\mathrm{HAY} \mathrm{Y}^{50}$ & $8-12$ & Inglés & 32 & $\begin{array}{l}\text { Síntomas, emociones, auto- } \\
\text { concepto y auto-manejo }\end{array}$ & $\begin{array}{l}\text { Autoadministrable al niño/ } \\
\text { adolescente }\end{array}$ & No \\
\hline ITG-CASF51,52 & $2-17$ & Inglés & 8 & $\begin{array}{l}\text { Síntomas diurnos, sínto- } \\
\text { mas nocturnos y limitación } \\
\text { funcional }\end{array}$ & $\begin{array}{l}\text { Autoadministrable a cuida- } \\
\text { dores }\end{array}$ & No \\
\hline
\end{tabular}

DMCR: Diferencia mínima clínicamente relevante, PAQLQ: Paediatric Asthma Quality of Life Questionnaire, PACQLQ: Paediatric Asthma Caregiver's Quality of Life Questionnaire, CHSA: Children's Health Survey for Asthma, CHSA-C: Children's Health Survey for AsthmaChild Version, PedsQL-AM 3.0: Pediatric Quality of Life Inventory Asthma Module 3.0, Pictorial PAQLQ: PAQLQ ilustrado, Mini PAQLQ: PAQLQ abreviado, AMA: About My Asthma, AAQOL: Adolescent Asthma Quality of Life Questionnaire, CAQ (A, B, C): Childhood Asthma Questionnaires (A,B,C Format), ARQOLS: Asthma-Related Quality of Life Scale, TACQOL-asthma: TNO-AZL Child Quality of Life of asthma, LAQCL: Life Activities Questionnaire for Childhood Asthma, HAY: How Are You?, ITG-CASF: Integrated Therapeutics Group Child Asthma Short Form. 
años podrían ser otra alternativa, si en el futuro fueran validadas en español. En calidad de vida el PAQLQ y PACQLQ son los más difundidos internacionalmente, siendo además los únicos con versiones en español, que ya han sido utilizadas en estudios clínicos de nuestro país.

\section{Referencias}

1.- Reddel H, Taylor D, Bateman E, et al: An offi cial American Thoracic Society/European Respiratory Society statement: asthma control and exacerbations: standardizing endpoints for clinical asthma trials and clinical practice. Am J Respir Crit Care Med 2009; 180: 59-99.

2.- Global Initiative for Asthma, Global Strategy for Asthma. Management and Prevention. Diagnosis and classification. Chapter 2. Updated 2011. Available at: http:// www.ginasthma.org [Consultado el 22 de septiembre de 2012].

3.- Pedersen S, Hurd S, Lemanske R, et al: Global strategy for the diagnosis and management of asthma in children 5 years and younger. Pediatr Pulmonol 2011; 46: 1-17.

4.- Gandhi R, Blaiss M: What are the best estimates of pediatric asthma control? Curr Opin Allergy Clin Immunol 2006; 6: 106-12.

5.- Cloutier M, Schatz M, Castro M, et al: Asthma outcomes: Composite scores of asthma control. J Allergy Clin Immunol 2012; 129: S24-33.

6.- Bime C, Nguyen J, Wise R: Measures of asthma control. Curr Opin Pulm Med 2012; 18:48-56.

7.- Bateman E, Bousquet J, Braunstein G: Is overall asthma control being achieved? A hypothesis-generating study. Eur Respir J 2001; 17: 589-95.

8.- Bateman E, Frith L, Braunstein G: Achieving guidelinebased asthma control: does the patient benefit?. Eur Respir J 2002; 20: 588-95.

9.- Bateman E, Hurd S, Barnes P, et al: Global strategy for asthma Management and prevention: GINA executive summary. Eur Respir J 2008; 31: 143-78.

10.- Nathan R, Sorkness C, Kosinski M, et al: Development of the asthma control test: a survey for assessing asthma control. J Allergy Clin Immunol 2004; 113: 59-65.

11.- Schatz M, Sorkness C, Li J, et al: Asthma Control Test: Reliability, validity, and responsiveness in patients not previously followed by asthma specialists. J Allergy Clin Immunol 2006; 117: 549-56.

12.- Vega J, Badia X, Badiola C, et al: Validation of the spanish versión of the asthma control test (ACT). J Asthma 2007; 44: 867-72.
13.- Vidal A, Ubilla C, Duffau D: Control de asma en adolescentes. Rev Med Chile 2008; 136: 859-66.

14.- Liu A, Zeiger R, Sorkness C, et al: Development and cross-sectional validation of the Childhood Asthma Control Test J Allergy Clin Immunol 2007; 119: 817-25.

15.- Juniper E, Gruffydd-Jones K, Ward S, Svensson K: Asthma Control Questionnaire in children: validation, measurement properties, interpretation. Eur Respir J 2010; 36: 1410-6.

16.- Skinner E, Diette G, Algatt-Bergstrom P: The Asthma Therapy Assessment Questionnaire (ATAQ) for children and adolescents. Dis Manag 2004; 7: 305-13.

17.- Diette G, Sajjan S, Skinner E, Weiss T, Wu A, Markson $L$ : Using the Pediatric Asthma Therapy Assessment Questionnaire to Measure Asthma Control and Healthcare Utilization in Children. Patient 2009; 2: 233-41.

18.- Pérez-Yarza E, Badia X, Badiola C, et al: Development and Validation of a Questionnaire to Assess Asthma Control in Pediatrics. Pediatr Pulmonolol 2009, 44: 5463.

19.- Murphy K, Zeiger R, Kosinski M, et al: Test for respiratory and asthma control in kids (TRACK): a caregivercompleted questionnaire for preschool-aged children. J Allergy Clin Immunol 2009; 23: 833-39.

20.- Chipps B, Zeiger O, Murphy K, et al: Longitudinal Validation of the Test for Respiratory and Asthma Control in Kids in Pediatric Practices. Pediatrics 2011; 127: 737-47.

21.- Ducharme F, Davis G, Noya F, Rich H, Ernst P: The Asthma Quiz for Kidz: a validated tool to appreciate the level of asthma control in children. Can Respir J 2004; 11: 541-6.

22.- Zorc J, Pawlowski N, Allen J, et al: Development and validation of an instrument to measure asthma symptom control in children. J Asthma 2006; 43: 753-8.

23.- Kachru R, Morphew T, Kehl S, et al: Validation of a single survey that can be used for case identification and assessment of asthma control: the Breathmobile Program. Ann Allergy Asthma Immunol 2006; 97: 77583.

24.- Rosier M, Bishop J, Nolan T, Robertson C, Carlin J, Phelan P: Measurement of functional severity of asthma in children. Am J Respir Crit Care Med 1994; 149 1434-41.

25.- Lara M, Sherbourne C, Duan N, Morales L, Gergen P, Brook R: An English and Spanish Pediatric Asthma Symptom Scale. Med Care 2000; 38: 342-50.

26.- Hallstrand T, Martin D, Hummel J, Williams B, Lo Gerfo J: Initial test of the seattle asthma severity and control questionnaire: a multidimensional assessment 
of asthma severity and control. Ann Allergy Asthma Immunol 2009; 103: 225-32.

27.- Thomas M, Gruffydd-Jones K, Stonham C, Ward S, Macfarlane T: Assessing asthma control in routine clinical practice: use of the Royal College of Physicians '3 questions'. Prim Care Respir J 2009; 18: 83-8.

28.- Quittner A, Modi A, Cruz I: Systematic review of health-related quality of life measures for children with respiratory conditions. Paediatr Respir Rev 2008; 9: 220-32.

29.- Wilson S, Rand C, Cabana M, et al: Asthma outcomes: Quality of life. J Allergy Clin Immunol 2012; 129: S88123.

30.- Juniper E, Guyatt G, Feeny D, Ferrie P, Griffith L, Townsend M: Measuring quality of life in children with asthma. Qual Life Res 1996; 5: 35-46.

31.- Juniper E, Guyatt G, Feeny D, Ferrie P, Griffith L, Townsend M: Measuring quality of life in the parents of children with asthma. Qual Life Res 1996; 5: 27-34.

32.- Measurement of Health-Related Quality of Life \& Asthma Control. Available at: http://www.qoltech.co.uk/ [Consultado el 16 de julio de 2013].

33.- Barrueto L, Yáñez M, Gálvez V, Mallol J: Calidad de vida en madres de lactantes portadores de sibilancias recurrentes. Rev Chil Enf Respir 2004; 20: 71-5.

34.- Vidal A, Duffau G, Ubilla C: Calidad de vida en el niño asmático y su cuidador. Rev Chil Enf Respir 2007; 23: 160-6.

35.- Velástegui C, Pérez-Canto P, Zárate V, et al: Impacto del asma en escolares de dos centros de salud primaria. Rev Med Chile 2010; 138: 205-12.

36.- Asmussen L, Olsen L, Grant E, Fagan J, Weiss K: Reliability and validity of the children's health survey for asthma. Pediatrics 1999; 104: 1-10.

37.- Olson L, Radecki L, Frintner $M$, et al: what age can children report dependably on their asthma health status? Pediatrics 2007; 119: 93-102.

38.- Radecki L, Olson L, Frintner M, Weiss K: Reliability and Validity of the Children's Health Survey for Asthma-Child Version. Ped Asthma Allergy Immunol 2008; 21: 89-98.

39.- Varni J, Burwinkle T, Rapoff M, Kamps J, Olson N: The PedsQL in pediatric asthma: reliability and validity of the pediatric quality of life inventory generic core scales and asthma module. J Behav Med 2004; 27: 297-318.

40.- Greenley R, Josie K, Drotar D: Self-reported quality of life among inner-city youth with asthma: an empirical examination of the PedsQL 3.0 Asthma Module. Ann
Allergy Asthma Immunol 2008; 100: 106-11.

41.- Seid M, Limbers C, Driscoll K, Opipari-Arrigan L, Gelhard L, Varni J: Reliability, validity, and responsiveness of the pediatric quality of life inventory (PedsQL) generic core scales and asthma symptoms scale in vulnerable children with asthma. J Asthma 2010; 47: 170-7.

42.- Everhart R, Fiese B: Development and initial validation of a pictorial quality of life measure for young children with asthma. J Pediatr Psychol 2009; 34: 966-76.

43.- Wing A, Upton J, Svensson K, Weller P, Fletcher M, Walker $S$ : The standardized and mini versions of the PAQLQ are valid, reliable, and responsive measurement tools. J Clin Epidemiol 2012; 65: 643-50.

44.- Mishoe S, Baker R, Poole S, Harrell L, Arant C, Rupp $N$ : Development of an instrument to assess stress levels and quality of life in children with asthma. J Asthma 1998; 35: 553-63.

45.- Rutishauser C, Sawyer S, Bond L, Coffey C, Bowes G: Development and validation of the Adolescent Asthma Quality of Life Questionnaire (AAQOL). Eur Respir J 2001; 17: 52-8.

46.- French D, Christie M, Sowden A: The reproducibility of the Childhood Asthma Questionnaires: Measures of quality of life for children with asthma aged 4-16 years. Qual Life Res 1994; 3: 215-24.

47.- Chiang L, Tzeng L, Fu L, Huang J: Testing a questionnaire to measure asthma-related quality of life among children. J Nurs Scholarsh 2006; 38: 383-86.

48.- Flapper B, Koopman H, Napel C, van der Schans C: Psychometric properties of the TACQOL-asthma, a disease-specific measure of health related quality of life for children with asthma and their parents. Chron Respir Dis 2006; 3: 65-72.

49.- Creer T, Wigal J, Kotses H, Hatala J, McConnaughy K, Winder J: A life activities questionnaire for childhood asthma. J Asthma 1993; 30: 467-73.

50.- le Coq E, Boeke A, Bezemer P, Colland V, van Eijk J: Which source should we use to measure quality of life in children with asthma: The children themselves or their parents? Qual Life Res 2000; 9: 625-36.

51.- Bukstein D, McGrath M, Buchnew D, Landgraf J, Goss $T$ : Evaluation of a short form for measuring healthrelated quality of life among pediatric asthma patients. J Allergy Clin Immunol 2000; 105: 245-51.

52.- Gorelick M, Brousseau D, Stevens M: Validity and responsiveness of a brief, asthma-specific quality of life instrument in children with acute asthma. Ann Allergy Asthma Immunol 2004; 92: 47-51. 